\title{
AVALIAÇÃO DE PROPRIEDADES FÍSICAS E MECÂNICAS DE MADEIRAS DE JATOBÁ (Hymenaea stilbocarpa Hayne) COM DIFERENTES TEORES DE UMIDADE E EXTRAÍdAS DE REGIÕES DISTINTAS ${ }^{1}$
}

\author{
Francisco Antonio Rocco Lahr², André Luis Christoforo ${ }^{3 *}$, Carlos Eduardo Gomes da Silva ${ }^{4}$, Jairo Ribas \\ de Andrade Junior ${ }^{5}$ e Roberto Vasconcelos Pinheiro ${ }^{6}$
}

\footnotetext{
${ }^{1}$ Recebido em 03.01.2015 aceito para publicação em 20.11.2015.

${ }^{2}$ Universidade de São Paulo, Escola de Engenharia de São Carlos, São Carlos, SP - Brasil. E-mail: <frocco@sc.usp.br>.

${ }^{3}$ Universidade Federal de São Carlos, São Carlos, SP - Brasil. E-mail: <christoforoal@yahoo.com.br>.

${ }^{4}$ Centro Universitário da Fundação Educacional de Barretos, Barretos, SP - Brasil. E-mail: <cegomes56@gmail.com>.

${ }^{5}$ Universidade de São Paulo, Escola de Engenharia de São Carlos, Programa de Pós-graduação em Engenharia de Estruturas. São Carlos, SP - Brasil. E-mail: <jairoribas@usp.br>.

${ }^{6}$ Universidade do Estado de Mato Grosso. Sinop, MT - Brasil. E-mail: <rpinheiro@unemat-net.br>.

${ }^{*}$ Autor para correspondência.
}

\begin{abstract}
RESUMO - Este trabalho objetivou investigar a influência das regiões de extração (Municípios de Caracaraí e Bonfim, Sul de Roraima; Município de Alta Floresta, Norte de Mato Grosso) da madeira da espécie jatobá (Hymenaea stilbocarpa). Também, foi avaliada a influência do teor de umidade (madeira saturada e a $12 \%$ ) em 15 propriedades físicas e mecânicas de interesse. Ao todo, foram preparados e ensaiados, segundo as prescrições da Norma Brasileira ABNT NR 7190 (1997), 840 corpos de prova. Os resultados da análise de variância (a 5\% de significância) revelaram que a região de extração dos corpos de prova não foi significativa nas propriedades físicas e mecânicas avaliadas, pois foram obtidos valores equivalentes, independentemente da origem, aspecto altamente interessante à medida que nem sempre é possível identificar a região de onde provém a madeira tropical. Essa importância é ainda maior no caso do emprego da madeira como elemento, cujos valores de referência se encontram apontados nos anexos do referido documento normativo. Com relação ao teor de umidade, foi observada diferença significativa em oito das 15 propriedades avaliadas. A condição de $12 \%$ de teor de umidade apresentou valores médios superiores à condição saturada nas propriedades de resistência na compressão paralela (37\%), resistência na tração paralela (29\%) e cisalhamento (36\%) na direção paralela às fibras; módulo de resistência na flexão (26\%), módulo de elasticidade na compressão paralela (9\%) e 200 direções paralelas $(35 \%)$ e normal (28\%) às fibras; e inferior na densidade aparente.
\end{abstract}

Palavras-chave: Madeira, Teor de umidade, Região de origem.

\section{EVALUATION OF PHYSICAL AND MECHANICAL PROPERTIES OF JATOBA (Hymenaea stilbocarpa Hayne) WOOD WITH DIFFERENT LEVELS OF MOISTURE CONTENT AND DIFFERENT REGIONS OF EXTRACIONS}

\footnotetext{
ABSTRACT - This research aimed to investigate the influence of the extraction regions (Caracari and Bonfim municipalities - South of Roraima; Alta Floresta municipality-North of Mato Grosso) of Jatobá (Hymenaea stilbocarpa) wood. The moisture content (saturated wood at 12\%) was also evaluated in fifteen physical and mechanical properties of interest. Altogether, 840 samples were prepared and tested, according to the requirements of the Brazilian standard ABNT NBR 7190. ANOVA results (at 5\% significance level) showed that the extraction region of the samples was not significant in the physical and mechanical properties evaluated, since equivalent values were obtained regardless of location, highly interesting
} 
aspect to the extent that it is not always possible to identify the extraction region of the tropical hardwood. This importance is even greater in the case of the use of wood as an element, the reference values of which are pointed out in the Annexes of the Brazilian standard. With respect to the moisture content, a significant difference was noted in only eight of the fifteen evaluated properties. The condition of $12 \%$ of moisture content showed mean values higher than the saturated condition in the strength properties in the parallel compression $(37 \%)$, resistance in parallel tension (29\%) and shear (36\%) strength in the direction parallel to the grain, bending strength (26\%), elasticity modulus in parallel compression (9\%) and 200 parallel directions (35\%) and normal (28\%) directions to the grain, and lower in bulk density.

Keywords: Wood, Moisture content, Region of origin.

\section{INTRODUÇÃO}

As madeiras da mesma espécie plantadas em regiões distintas estão sujeitas a variações de suas propriedades físicas e mecânicas (PLETZ et al., 2006), decorrentes dos estresses sofridos, assim como por fatores edafoclimáticos durante seu crescimento, inerentes ao local e região onde essas madeiras foram cultivadas (RODRIGUES et al., 2008).

As espécies arbóreas brasileiras despertam o interesse de vários pesquisadores para demonstrar sua adaptação, o que potencializa a utilização da madeira em áreas até então inóspitas para outras espécies. $\mathrm{O}$ jatobá (Hymenaea courbaril) tem vasta distribuição geográfica e habilidade em se desenvolver bem em ambientes com diferentes características edafoclimáticas, destacando-se no cenário científico nacional por apresentar estratégias adaptativas interessantes (PAIVA, 2003; NASCIMENTO, 2011).

A espécie jatobá (Hymenaea spp.) é encontrada em quase todas as matas nativas do Brasil. A espécie Hymenaea stilbocarpa Hayne ocorre desde o Estado do Piauí até o Paraná, sendo a espécie Hymenaea courbaril L. mais comum na Amazônia. Essas madeiras têm características semelhantes para densidade e caracteres anatômicos, sendo tratadas em conjunto na descrição de suas propriedades (IPT, 1989). Segundo Rizzini (1978), a espécie é conhecida no Brasil por jatobá, jutaí, jataí, burandá, castanheiro-de-bugre, courbaril, farinheira, jataí-ibá, jati, jataí-amarelo, jataí-vermelho etc.; na Argentina, por "paqui" e "copaíba".

Conhecer as propriedades físicas e mecânicas possibilita um uso mais racional da madeira (CARREIRA et al., 2012; MINÁ; DIAS, 2008). A Norma Brasileira ABNT NBR7190 (1997) estabelece a caracterização completa para espécies não conhecidas e determina as propriedades físicas e mecânicas na condição-padrão do teor de umidade de 12\% (DIAS; LAHR, 2004): resistência à compressão paralela às fibras; resistência à compressão e à tração normal às fibras; resistência ao cisalhamento paralelo às fibras; resistência ao embutimento paralelo e normal às fibras; densidade básica; e densidade aparente.

O Anexo B da norma ABNT NBR 7190 (1997) estabelece os ensaios das propriedades citadas e apresenta também os ensaios para obtenção do módulo de elasticidade longitudinal na compressão e tração paralela às fibras; módulo de elasticidade no ensaio de flexão estática; tenacidade; entre outras propriedades.

As propriedades físicas de referência para as madeiras de jatobá, determinadas pelo Instituto de Pesquisas Tecnológicas (IPT, 1989), caracterizam-na como espécie altamente resistente ao ataque de fungos, impermeável a tratamentos preservativos, com densidade aparente de $0,96 \mathrm{~g} / \mathrm{cm}^{3}$ a $15 \%$ de teor de umidade e densidade básica de $0,80 \mathrm{~g} / \mathrm{cm}^{3}$. As propriedades mecânicas são elevadas, o valor médio de resistência na compressão paralela às fibras $\left(\mathrm{f}_{\mathrm{c} 0}\right)$ são de $82,2 \mathrm{MPa}$ (a 15\% de teor de umidade) e $67 \mathrm{MPa}$ na madeira em condição saturada. $\mathrm{N}$ a madeira saturada, o módulo de elasticidade médio na flexão é de 14,84 MPa e de $17,69 \mathrm{MPa}$ na compressão, retração radial de $3,1 \% \mathrm{e}$ volumétrica de $10,7 \%$, resistência média ao cisalhamento na direção paralela $\left(\mathrm{f}_{\mathrm{v} 0}\right)$ de $17,5 \mathrm{MPa}$, fendilhamento $\left(f_{s}\right)$ de 1,5 MPa e tração normal às fibras $\left(f_{t 90}\right)$ de 13,1 $\mathrm{MPa}$.

As densidades das madeiras de jatobá variam, além de outros fatores, em função da espécie. Segundo Simpson (1996), a massa específica damadei ra de Hymenaea oblongifolia é de $0,74 \mathrm{~g} / \mathrm{cm}^{3}$ e de $0,71 \mathrm{~g} / \mathrm{cm}^{3}$ e de 0,76 $\mathrm{g} / \mathrm{cm}^{3}$ para a madeira de Hymenaea courbaril, segundo as pesquisas de Bergman et al. (1999) e Souza et al. (1997), respectivamente. 
Dias e Lahr (2004) enquadraram madeiras de jatobá na classe de resistência C60 da Norma Brasileira ABNT NBR 7190 (1997), por apresentarem valor característico da resistência na compressão de 78,7 MPa. As demais características mecânicas obtidas foram: resistência na flexão estática de $159 \mathrm{MPa}$, módulo de elasticidade na compressão paralela às fibras de $22,967 \mathrm{MPa}$, módulo de elasticidade na tração paralela às fibras de 21,39 $\mathrm{MPa}$, módulo de elasticidade na flexão estática de 21,36 $\mathrm{MPa}$, dureza paralela às fibras $\left(\mathrm{f}_{\mathrm{H} 0}\right)$ de $165 \mathrm{MPa}$, dureza normal às fibras $\left(\mathrm{f}_{\mathrm{H} 90}\right)$ de $127 \mathrm{MPa}$ e tenacidade $(\mathrm{W})$ 2,11 da N.m.

Longsdon (1998) estudou a influência do teor de umidade da madeira de jatobá em suas propriedades de resistência e rigidez, com destaque para a compressão paralela às fibras, que é a característica adotada pela Norma BrasileiraABNT NBR 7190 (1997) para categorizar as espécies em classes de resistência. $\mathrm{O}$ aumento progressivo do teor de umidade foi responsável por redução significativa da resistência na compressão para níveis variando de $0 \%$ a valores próximos de $20 \%$, mantendo-se praticamente constante para teores de umidade entre $30 \%$ e $50 \%$ e também pelo aumento progressivo na densidade das madeiras.

Uma variável que também pode influenciar nas propriedades físicas e mecânicas da madeira consiste na região de extração das árvores, assim como discutido nas pesquisas de Lima (2010) e Moraes Neto et al. (2009).

Lima (2010) utilizou teste de procedência sobre o comportamento silvicultural de populações da espécie Gallesia integrifolia (pau-d'alho) e estudou o efeito das procedências em propriedades da madeira, com mudas de três procedências (Bauru, Ribeirão Preto e Campinas), que foram plantadas em Luiz Antônio, SP, com idade de 26 anos do plantio. As propriedades estudadas foram: densidade aparente, densidade básica, resistência à compressão paralela às fibras, retração volumétrica e coeficiente de anisotropia. Esse autor concluiu que a procedência das sementes influenciou significativamente a densidade aparente, enquanto a resistência à compressão paralela às fibras, densidade básica, retração volumétrica e coeficiente de anisotropia não apresentaram diferenças significativas pelo efeito de diferentes procedências das sementes.

Moraes Neto et al. (2009) analisaram as variações das propriedades físicas e mecânicas da madeira de cinco procedências de Pinus caribaea var. Hondurensis da América Central, implantadas no Cerrado do Distrito Federal, extraídas com idade de 23 anos. As propriedades físicas da madeira estudadas foram a densidade e a estabilidade dimensional (contração e inchamento em função do teor de umidade), e as propriedades mecânicas foram a resistência à compressão, flexão, tração, cisalhamento e fendilhamento. Não foram observadas diferenças significativas em todas as características analisadas.

Em linhas gerais, as madeiras de jatobá têm sido foco de estudos realizados por diversos autores, entretanto não tendo sido investigada a influência de diferentes regiões de extração das madeiras de jatobá nas suas propriedades físicas e mecânicas, fator que se mostra importante, uma vez que as madeiras obtidas para a construção de estruturas, entre outras aplicações, podem ser advindas de regiões diversas. Este trabalho objetivou investigar, com o auxílio da análise de variância (ANOVA), a influência da região de extração das madeiras de jatobá (três regiões distintas), assim como dos teores de umidade (12\% e saturada) em algumas propriedades físicas e mecânicas investigadas.

\section{MATERIAL E MÉTODOS}

As propriedades investigadas nesta pesquisa foram:

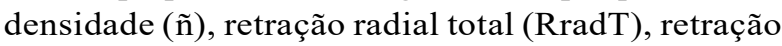
tangencial total $(\mathrm{R} \operatorname{tgT})$, resistência na compressão paralela às fibras $\left(\mathrm{f}_{\mathrm{c} 0}\right)$, resistência na tração paralela $\left(\mathrm{f}_{\mathrm{t} 0}\right)$, resistência na tração perpendicular às fibras $\left(\mathrm{f}_{t 90}\right)$, resistência ao cisalhamento na direção paralela $\left(\mathrm{f}_{\mathrm{v} 0}\right)$, resistência ao fendilhamento $\left(\mathrm{f}_{\mathrm{s} 0}\right)$, resistência na flexão $\left(\mathrm{f}_{\mathrm{m}}\right)$, módulo de elasticidade na compressão paralela $\left(\mathrm{E}_{\mathrm{c} 0}\right)$, módulo de elasticidade na tração paralela $\left(\mathrm{E}_{\mathrm{t} 0}\right)$, módulo de elasticidade na flexão $\left(\mathrm{E}_{\mathrm{m}}\right)$, dureza na direção paralela $\left(\mathrm{f}_{\mathrm{H} 0}\right)$ e perpendicular $\left(\mathrm{f}_{\mathrm{H} 90}\right)$ às fibras e tenacidade (W), ambas obtidas seguindo as premissas e métodos de cálculo da norma brasileira ABNT NBR 7190 (1997).

Os ensaios foram realizados nas dependências do Laboratório de Madeiras e de Estruturas de Madeira (LaMEM), do Departamento de Engenharia de Estruturas da Escola de Engenharia de São Carlos, da Universidade de São Paulo, em São Paulo.

Por propriedade física e mecânica e para os dois teores de umidade ( $12 \%$ e saturado), foram utilizadas 10 amostras de madeiras de jatobá obtidas no Município de Caracari' do Sul, de Roraima [SRo-A]; 13 amostras

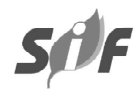

Revista Árvore, Viçosa-MG, v.40, n.1, p.147-154, 2016 
advindas do Município de Bonfim do Sul, de Roraima [SRo-B]; e 12 corpos de prova obtidos do Município Alta Floresta, do Norte de Mato Grosso [N-MG], totalizando a confecção e experimentação de 840 amostras.

Para investigar a influência das regiões de extração das madeiras (SRo-A; SRo-B; N-MG]), assim como do teor de umidade ( $12 \%$ e saturado) nas propriedades físicas e mecânicas investigadas, foi utilizada a análise de variância a $5 \%$ de significância $(a ́)$, tendo a equivalência entre os valores médios das propriedades como hipótese nula $\left(H_{0}\right)$ e a não equivalência como hipótese alternativa $\left(H_{l}\right)$. P-valor inferior ao nível de significância implica rejeitar $H_{0}$, aceitando-a em caso contrário. $\mathrm{O}$ teste de comparações múltiplas de Tukey foi utilizado para o devido agrupamento dos níveis dos fatores.

Para validação da ANOVA, foram avaliadas a normalidade (teste de Anderson-Darling) na distribuição das respostas e a homogeneidade de variâncias entre os níveis do fator (teste de Bartlett). Ambos os testes foram considerados a $5 \%$ de significância. O teste de Anderson-Darling teve como hipótese nula a normalidade nas distribuições e a não normalidade como hipótese alternativa. P-valor superior ao nível de significância implica aceitar $H_{0}$, refutando-a em caso contrário. $\mathrm{O}$ teste de Bartlett teve a equivalência das variâncias entre os tratamentos como hipótese nula e a não equivalência como hipótese alternativa. P-valor superior ao nível de significância implica aceitar $H_{0}$, refutando-a em caso contrário.

\section{RESULTADOS}

As Tabelas 1 e 2 apresentam os resultados médios $(\bar{x})$ e os coeficientes de variação $(C v)$ das propriedades físicas e mecânicas investigadas nas madeiras de jatobá com teor de umidade de $12 \%$ e saturada, respectivamente.

As Tabelas 3 e 4 apresentam os resultados dos testes de normalidade (Anderson-Darling [A-D]) e de homogeneidade entre variâncias (Bartlett), da análise de variância e do teste de comparações múltiplas de Tukey para as propriedades (Prop.) físicas e mecânicas das madeiras de jatobá com teor de umidade de $12 \%$ e saturada, respectivamente. Nas Tabelas 3 e 4, GL denota os graus de liberdade da ANOVA e letras iguais implicam tratamentos com médias equivalentes.

A Tabela 5 apresenta os resultados dos testes de normalidade e homogeneidade entre variâncias, da análise de variância e do teste de comparações múltiplas de Tukey (valores médios e de agrupamento) para as propriedades físicas e mecânicas das madeiras de jatobá para os dois teores de umidade, encontrando-se sublinhados os P-valores da ANOVA considerados significativos. Ressalta-se que, como não houve diferença significativa das posições de extração das madeiras, as possíveis variações encontradas são explicadas pelas diferenças no teor de umidade. Para essa análise, foram agrupados, por teor de umidade, os resultados das experimentações com as madeiras de jatobá das três localidades investigadas por resposta.

Tabela 1 - Resultados das propriedades físicas e mecânicas das madeiras de jatobá a $12 \%$ de umidade. Table 1 -Results of physical and mechanical properties of jatobá wood with $12 \%$ moisture content.

\begin{tabular}{|c|c|c|c|c|c|c|}
\hline \multirow[t]{2}{*}{ Propriedades } & \multicolumn{2}{|c|}{ SRo-A } & \multicolumn{2}{|c|}{ SRo-B } & \multicolumn{2}{|c|}{ S-Pará } \\
\hline & $x$ & $C v(\%)$ & $x$ & $C v(\%)$ & $x$ & $C v(\%)$ \\
\hline$\rho(\mathrm{g} / \mathrm{cm} 3)$ & 1,05 & 11 & 1,08 & 8 & 0,91 & 13 \\
\hline RradT (\%) & 3,52 & 11 & 3,63 & 18 & 3,45 & 15 \\
\hline RtgT (\%) & 7,23 & 8 & 6,71 & 10 & 6,63 & 8 \\
\hline $\mathrm{f}_{\mathrm{c} 0}(\mathrm{MPa})$ & 93,91 & 7 & 93,42 & 11 & 94,38 & 8 \\
\hline $\mathrm{f}_{\mathrm{t} 0}^{\mathrm{co}}(\mathrm{MPa})$ & 167,8 & 16 & 141,33 & 19 & 153,46 & 19 \\
\hline $\mathrm{f}_{\mathrm{t} 90}(\mathrm{MPa})$ & 4,13 & 19 & 3,71 & 23 & 3,23 & 17 \\
\hline $\mathrm{f}_{\mathrm{v} 0}(\mathrm{MPa})$ & 24,70 & 18 & 28,00 & 21 & 26,69 & 16 \\
\hline $\mathrm{f}_{\mathrm{s} 0}(\mathrm{MPa})$ & 0,86 & 20 & 0,85 & 19 & 0,82 & 21 \\
\hline $\mathrm{f}_{\mathrm{m}}^{\mathrm{s}}(\mathrm{MPa})$ & 163,42 & 23 & 151,83 & 16 & 148,46 & 19 \\
\hline $\mathrm{E}_{\mathrm{c} 0}(\mathrm{MPa})$ & 22482 & 21 & 21403 & 14 & 21759 & 19 \\
\hline $\mathrm{E}_{\mathrm{to}}(\mathrm{MPa})$ & 22195 & 6 & 20801 & 7 & 21752 & 11 \\
\hline $\mathrm{E}_{\mathrm{m}}(\mathrm{MPa})$ & 22708 & 11 & 20864 & 8 & 21222 & 10 \\
\hline $\mathrm{f}_{\mathrm{HO}}(\mathrm{MPa})$ & 160,33 & 7 & 166,17 & 8 & 169,54 & 11 \\
\hline $\mathrm{f}_{\mathrm{H} 90}(\mathrm{MPa})$ & 133 & 6 & 128,67 & 10 & 141,08 & 16 \\
\hline $\mathrm{W}(\mathrm{N} \cdot \mathrm{m})$ & 18,45 & 13 & 21,67 & 13 & 19,82 & 19 \\
\hline
\end{tabular}


Tabela 2 - Resultados das propriedades físicas e mecânicas das madeiras de jatobá na condição saturada. Table 2 - Results of physical and mechanical properties of jatobá wood in satured condition.

\begin{tabular}{|c|c|c|c|c|c|c|}
\hline \multirow[t]{2}{*}{ Propriedades } & \multicolumn{2}{|c|}{ SRo-A } & \multicolumn{2}{|c|}{ SRo-B } & \multicolumn{2}{|c|}{ S-Pará } \\
\hline & $x$ & $C v(\%)$ & $x$ & $C v(\%)$ & $x$ & $C v(\%)$ \\
\hline$\rho(\mathrm{g} / \mathrm{cm} 3)$ & 1,21 & 4 & 1,21 & 5 & 1,09 & 4 \\
\hline $\operatorname{RradT}(\%)$ & 3,52 & 11 & 3,52 & 21 & 3,55 & 11 \\
\hline $\operatorname{RtgT}(\%)$ & 7,20 & 8 & 6,59 & 12 & 6,72 & 5 \\
\hline $\mathrm{f}_{\mathrm{c} 0}(\mathrm{MPa})$ & 69,52 & 5 & 64,23 & 21 & 72,58 & 10 \\
\hline $\mathrm{f}_{\mathrm{t} 0}(\mathrm{MPa})$ & 127,61 & 19 & 108,46 & 26 & 115,92 & 20 \\
\hline $\mathrm{f}_{\mathrm{t} 90}(\mathrm{MPa})$ & 3,54 & 23 & 2,35 & 18 & 3,3 & 16 \\
\hline $\mathrm{f}_{\mathrm{v} 0}(\mathrm{MPa})$ & 18,31 & 10 & 19,54 & 13 & 20,08 & 23 \\
\hline $\mathrm{f}_{\mathrm{s} 0}(\mathrm{MPa})$ & 0,82 & 19 & 0,89 & 16 & 0,72 & 19 \\
\hline $\mathrm{f}_{\mathrm{m}}(\mathrm{MPa})$ & 126,34 & 16 & 119,15 & 18 & 116,08 & 16 \\
\hline $\mathrm{E}_{\mathrm{c} 0}(\mathrm{MPa})$ & 22980 & 19 & 19318 & 15 & 20196 & 21 \\
\hline $\mathrm{E}_{\mathrm{t} 0}(\mathrm{MPa})$ & 20918 & 11 & 20112 & 8 & 21617 & 11 \\
\hline $\mathrm{E}_{\mathrm{m}}(\mathrm{MPa})$ & 22295 & 15 & 18809 & 10 & 20854 & 10 \\
\hline $\mathrm{f}_{\mathrm{H} 0}(\mathrm{MPa})$ & 107,20 & 23 & 119,85 & 19 & 128,75 & 18 \\
\hline $\mathrm{f}_{\mathrm{H} 90}(\mathrm{MPa})$ & 97,92 & 12 & 105,62 & 19 & 110,92 & 11 \\
\hline $\mathrm{W}(\mathrm{N} \cdot \mathrm{m})$ & 16,85 & 21 & 20,12 & 22 & 16,39 & 19 \\
\hline
\end{tabular}

Tabela 3 - Resultados da ANOVA e do teste de Tukey para as propriedades físicas e mecânicas das madeiras de jatobá a $12 \%$ de umidade.

Table $3-A N O V A$ and Tukey test results for the physical and mechanical properties of jatobá wood with $12 \%$ moisture content.

\begin{tabular}{|c|c|c|c|c|c|c|c|}
\hline \multirow[t]{2}{*}{ Prop. } & \multicolumn{2}{|c|}{ P-valor } & \multicolumn{2}{|c|}{ ANOVA } & \multicolumn{3}{|c|}{ Tukey (Agrupamento) } \\
\hline & A-D & Bartlett & GL & P-valor & SRo-A & SRo-B & $\mathrm{N}-\mathrm{MG}$ \\
\hline$\rho\left(\mathrm{g} / \mathrm{cm}^{3}\right)$ & 0,127 & 0,388 & 34 & 0,371 & $\mathrm{~A}$ & $\mathrm{~A}$ & A \\
\hline $\operatorname{RradT}(\%)$ & 0,245 & 0,352 & 34 & 0,988 & A & A & A \\
\hline $\operatorname{RtgT}(\%)$ & 0,132 & 0,302 & 34 & 0,070 & A & A & A \\
\hline $\mathrm{f}_{\mathrm{c} 0}(\mathrm{MPa})$ & 0,255 & 0,464 & 34 & 0,990 & A & A & A \\
\hline $\mathrm{f}_{\mathrm{t} 0}(\mathrm{MPa})$ & 0,398 & 0,605 & 34 & 0,100 & A & A & A \\
\hline $\mathrm{f}_{\mathrm{t} 90}(\mathrm{MPa})$ & 0,514 & 0,476 & 34 & 0,319 & A & A & A \\
\hline $\mathrm{f}_{\mathrm{v} 0}(\mathrm{MPa})$ & 0,174 & 0,339 & 34 & 0,129 & A & A & A \\
\hline $\mathrm{f}_{\mathrm{s} 0}(\mathrm{MPa})$ & 0,243 & 0,472 & 34 & 0,923 & A & A & A \\
\hline $\mathrm{f}_{\mathrm{m}}(\mathrm{MPa})$ & 0,212 & 0,621 & 34 & 0,236 & A & A & A \\
\hline $\mathrm{E}_{\mathrm{c} 0}(\mathrm{MPa})$ & 0,172 & 0,299 & 34 & 0,422 & A & A & A \\
\hline $\mathrm{E}_{\mathrm{t} 0}(\mathrm{MPa})$ & 0,411 & 0,553 & 34 & 0,285 & A & A & A \\
\hline $\mathrm{E}_{\mathrm{m}}(\mathrm{MPa})$ & 0,207 & 0,468 & 34 & 0,105 & A & A & A \\
\hline $\mathrm{f}_{\mathrm{H} 0}(\mathrm{MPa})$ & 0,358 & 0,427 & 34 & 0,369 & A & A & A \\
\hline $\mathrm{f}_{\mathrm{H} 90}(\mathrm{MPa})$ & 0,174 & 0,396 & 34 & 0,207 & A & A & A \\
\hline $\mathrm{W}(\mathrm{N} \cdot \mathrm{m})$ & 0,277 & 0,539 & 34 & 0,162 & A & A & A \\
\hline
\end{tabular}

\section{DISCUSSÕES}

Os valores da densidade das madeiras de jatobá encontrados nesta pesquisa (Tabelas 1 e 2) são superiores aos das pesquisas de Simpson (1996), Bergman et al. (1999) e Souza et al. (1997), que investigaram outras variedades de jatobá e próximos dos valores apresentados pelo Instituto de Pesquisas Tecnológicas (1989). Com relação às demais propriedades, os resultados deste estudo indicam comportamentos variados se comparados os das propriedades obtidas do trabalho de Dias e Lahr (2004) e do IPT (1989).

Os P-valores dos testes de normalidade e de homogeneidade entre variâncias (Tabelas 3 e 4) foram superiores a $5 \%$ em todos os casos, validando o modelo da ANOVA. Para todas as respostas investigadas, nas Tabelas 3 e 4 constata-se que a origem de extração das madeiras não foi significativa para os teores de umidade, resultado esse que está em conformidade 
Tabela 4 - Resultados da ANOVA e do teste de Tukey para as propriedades físicas e mecânicas das madeiras de jatobá da condição saturada.

Table 4-ANOVA and Tukey test results for the physical and mechanical properties of jatobá wood in saturated condition.

\begin{tabular}{|c|c|c|c|c|c|c|c|}
\hline \multirow[t]{2}{*}{ Prop. } & \multicolumn{2}{|c|}{ P-valor } & \multicolumn{2}{|c|}{ ANOVA } & \multicolumn{3}{|c|}{ Tukey (Agrupamento) } \\
\hline & A-D & Bartlett & GL & P-valor & SRo-A & SRo-B & N-MG \\
\hline$\rho\left(\mathrm{g} / \mathrm{cm}^{3}\right)$ & 0,264 & 0,452 & 34 & 0,263 & A & A & A \\
\hline RradT(\%) & 0,580 & 0,564 & 34 & 0,988 & A & A & A \\
\hline $\operatorname{RtgT}(\%)$ & 0,450 & 0,565 & 34 & 0,070 & A & A & A \\
\hline $\mathrm{f}_{\mathrm{c} 0}(\mathrm{MPa})$ & 0,507 & 0,703 & 34 & 0,109 & A & A & A \\
\hline $\mathrm{f}_{\mathrm{t} 0}(\mathrm{MPa})$ & 0,640 & 0,892 & 34 & 0,536 & A & A & A \\
\hline $\mathrm{f}_{t 90}(\mathrm{MPa})$ & 0,447 & 0,510 & 34 & 0,117 & A & A & A \\
\hline $\mathrm{f}_{\mathrm{v} 0}(\mathrm{MPa})$ & 0,342 & 0,704 & 34 & 0,313 & A & A & A \\
\hline $\mathrm{f}_{\mathrm{s} 0}(\mathrm{MPa})$ & 0,284 & 0,362 & 34 & 0,261 & A & A & A \\
\hline $\mathrm{f}_{\mathrm{m}}(\mathrm{MPa})$ & 0,129 & 0,636 & 34 & 0,098 & A & A & A \\
\hline $\mathrm{E}_{\mathrm{c} 0}(\mathrm{MPa})$ & 0,107 & 0,416 & 34 & 0,619 & A & A & A \\
\hline $\mathrm{E}_{\mathrm{t} 0}(\mathrm{MPa})$ & 0,158 & 0,323 & 34 & 0,219 & A & A & A \\
\hline $\mathrm{E}_{\mathrm{m}}(\mathrm{MPa})$ & 0,118 & 0,268 & 34 & 0,153 & A & A & A \\
\hline $\mathrm{f}_{\mathrm{H} 0}(\mathrm{MPa})$ & 0,275 & 0,513 & 34 & 0,422 & A & A & A \\
\hline $\mathrm{f}_{\mathrm{H} 90}(\mathrm{MPa})$ & 0,414 & 0,420 & 34 & 0,170 & A & A & A \\
\hline$\underline{\mathrm{W}}(\mathrm{N} \cdot \mathrm{m})$ & 0,236 & 0,646 & 34 & 0,309 & A & A & A \\
\hline
\end{tabular}

Tabela 5 - Resultados da ANOVA e do teste de Tukey das propriedades físicas e mecânicas para os teores de umidade de $12 \%$ e saturada.

Table 5-ANOVA and Tukey test results for the physical and mechanical properties of jatobá wood with 12\% moisture content and satured condition.

\begin{tabular}{|c|c|c|c|c|c|c|}
\hline \multirow[t]{2}{*}{ Prop. } & \multicolumn{2}{|c|}{ P-valor } & \multicolumn{2}{|c|}{ ANOVA } & \multicolumn{2}{|c|}{ Tukey (Agrupamento) } \\
\hline & A-D & Bartlett & GL & P-valor & $12 \%$ & Sat. \\
\hline$\rho\left(\mathrm{g} / \mathrm{cm}^{3}\right)$ & 0,686 & 0,875 & 69 & 0,000 & $\begin{array}{c}\mathrm{B} \\
1,00\end{array}$ & $\begin{array}{c}\mathrm{A} \\
1,17\end{array}$ \\
\hline $\operatorname{RradT}(\%)$ & 0,425 & 0,559 & 69 & 0,815 & $\begin{array}{c}\text { A } \\
3,48\end{array}$ & $\begin{array}{c}\text { A } \\
3,53\end{array}$ \\
\hline RtgT(\%) & 0,388 & 0,525 & 69 & 0,940 & $\begin{array}{c}\text { A } \\
6,78\end{array}$ & $\begin{array}{c}\text { A } \\
6,81\end{array}$ \\
\hline $\mathrm{f}_{\mathrm{co}}(\mathrm{MPa})$ & 0,411 & 0,576 & 69 & 0,000 & $\begin{array}{c}\text { A } \\
93,91\end{array}$ & $\begin{array}{c}\text { B } \\
68,60\end{array}$ \\
\hline $\mathrm{f}_{\mathrm{t} 0}(\mathrm{MPa})$ & 0,140 & 0,085 & 69 & 0,000 & $\begin{array}{c}\text { A } \\
153,40\end{array}$ & $\begin{array}{c}\text { B } \\
119,34\end{array}$ \\
\hline $\mathrm{f}_{\mathrm{t} 90}(\mathrm{MPa})$ & 0,199 & 0,495 & 69 & 0,189 & $\begin{array}{c}\text { A } \\
3,47\end{array}$ & $\begin{array}{c}\text { A } \\
3,16\end{array}$ \\
\hline $\mathrm{f}_{\mathrm{v} 0}(\mathrm{MPa})$ & 0,351 & 0,649 & 69 & 0,000 & $\begin{array}{c}\text { A } \\
26,28\end{array}$ & $\begin{array}{c}\text { B } \\
19,37\end{array}$ \\
\hline $\mathrm{f}_{\mathrm{s} 0}(\mathrm{MPa})$ & 0,124 & 0,373 & 69 & 0,602 & $\begin{array}{c}\text { A } \\
0,84\end{array}$ & $\begin{array}{c}\text { A } \\
0,81\end{array}$ \\
\hline $\mathrm{f}_{\mathrm{m}}(\mathrm{MPa})$ & 0,226 & 0,440 & 69 & 0,000 & $\begin{array}{c}\text { A } \\
157,11\end{array}$ & $\begin{array}{c}\text { B } \\
124,71\end{array}$ \\
\hline $\mathrm{E}_{\mathrm{co}}(\mathrm{MPa})$ & 0,202 & 0,670 & 69 & 0,013 & $\begin{array}{c}\text { A } \\
22589\end{array}$ & $\begin{array}{c}\text { B } \\
20666\end{array}$ \\
\hline $\mathrm{E}_{\mathrm{t} 0}(\mathrm{MPa})$ & 0,153 & 0,702 & 69 & 0,154 & $\begin{array}{c}\text { A } \\
21553\end{array}$ & $\begin{array}{c}\text { A } \\
20858\end{array}$ \\
\hline $\mathrm{E}_{\mathrm{m}}(\mathrm{MPa})$ & 0,072 & 1,152 & 69 & 0,077 & $\begin{array}{c}\text { A } \\
21524\end{array}$ & $\begin{array}{c}\text { A } \\
20506\end{array}$ \\
\hline $\mathrm{f}_{\mathrm{H} 0}(\mathrm{MPa})$ & 0,240 & 0,665 & 69 & 0,000 & $\begin{array}{c}\mathrm{A} \\
165,74\end{array}$ & $\begin{array}{c}\text { B } \\
122,71\end{array}$ \\
\hline $\mathrm{f}_{\mathrm{H} 90}(\mathrm{MPa})$ & 0,136 & 0,275 & 69 & 0,000 & $\begin{array}{c}\text { A } \\
134,51\end{array}$ & $\begin{array}{c}\text { B } \\
105,23\end{array}$ \\
\hline W (N.m) & 0,403 & 0,733 & 69 & 0,104 & $\begin{array}{c}\text { A } \\
4,24\end{array}$ & $\begin{array}{c}\text { A } \\
6,45\end{array}$ \\
\hline
\end{tabular}

Revista Árvore, Viçosa-MG, v.40, n.1, p.147-154, 2016 
com os obtidos do trabalho de Moraes Neto et al. (2009) e parcialmente com os da pesquisa de Lima (2010).

Das 15 propriedades investigadas (Tabela 5), o teor de umidade foi significativo em apenas oito. Das oito propriedades consideradas significativas pela ANOVA, a condição saturada apresentou valores superiores à condição de $12 \%$ de umidade apenas na densidade (17\%), fornecendo resultados inferiores nas sete demais. Os valores de $\mathrm{f}_{\mathrm{c} 0}, \mathrm{f}_{\mathrm{t} 0}, \mathrm{f}_{\mathrm{v} 0}, \mathrm{f}_{\mathrm{m}}, \mathrm{E}_{\mathrm{c} 0}, \mathrm{f}_{\mathrm{H} 0}$ e $\mathrm{f}_{\mathrm{H} 90}$ para o teor de umidade de $12 \%$ foram, em média, de $37 \%, 29 \%, 36 \%, 26 \%, 9 \%, 35 \%$ e $28 \%$ superiores a $f_{c 0}$, $\mathrm{f}_{\mathrm{t} 0}, \mathrm{f}_{\mathrm{v} 0}, \mathrm{f}_{\mathrm{m}}, \mathrm{E}_{\mathrm{c} 0}, \mathrm{f}_{\mathrm{H} 0}$ e $\mathrm{f}_{\mathrm{H} 90}$ das madeiras de jatobá na condição saturada. E isso evidencia as minerações significativas nas propriedades de resistência, comportamento esse similar ao encontrado na pesquisa de Longsdon (1998).

\section{CONCLUSÕES}

Os resultados encontrados revelaram que as propriedades físicas e mecânicas investigadas nas madeiras de jatobá nos dois teores de umidade foram equivalentes quanto ao local de extração, implicando não ser significativa a escolha da região na obtenção das respostas investigadas.

Com relação ao teor de umidade, notou-se diferença significativa apenas em oito das 15 propriedades avaliadas: densidade, resistências à compressão, tração e cisalhamento na direção paralela às fibras, resistência à flexão, módulo de elasticidade na compressão paralela e durezas paralela e normal às fibras. A condição saturada apresentou valor médio superior apenas na densidade, fornecendo valores significativamente inferiores nas sete demais propriedades.

\section{AGRADECIMENTOS}

À Fundação de Amparo à Pesquisa do Estado de São Paulo (FAPESP) e ao Conselho Nacional de Desenvolvimento Científico e Tecnológico (CNPq), pelo apoio financeiro, fundamental para o desenvolvimento desta pesquisa.

\section{REFERÊNCIAS}

ASSOCIAÇÃO BRASILEIRA DE NORMAS TÉCNICAS - ABNT. NBR 7190. Projeto de estruturas de madeira. Rio de Janeiro: 1997.
BERGMAN, R.; CAI, Z.; CARLL, C.G.; CLAUSEN, C.A.; DIETENBERGER, M.A.; FALK, R.H.; FRIHART, C.R.; GLASS, S.V.; HUNT, C.G.; IBACH, R.E.; KRETSCHMANN, D.E.; RAMMER, D.R.; ROSS, R.J. Wood Handbook - Wood as an engineering material. Madison: U.S. Department of Agriculture, Forest Service, Forest Products Laboratory, 1999. (Gen. Tech. Rep. FPLGTR-113)

CARREIRA, M.R.; SEGUNDINHO, P.G.A.; DIAS, A.A. Determination of the e/g ratio of wood logs using transverse vibration. Wood Research, v.57, p.207-220, 2012.

DIAS, F.M.; LAHR, F.A.R. Estimativa de propriedades de resistência e rigidez da madeira através da densidade aparente. Scientia Forestalis, n.65, p.102-113, 2004.

INSTITUTO DE PESQUISAS TECNOLÓGICAS DO ESTADO DE SÃO PAULO - IPT. Fichas de características das madeiras brasileiras. 2.ed. São Paulo: IPT, 1989. 418p. (IPT, 1791).

LIMA, I.L. Efeito da procedência em algumas propriedades da madeira de Gallesia integrifolia (Spreng.) Harms. Revista do Instituto Florestal, n.61, p.1-69, 2010.

LONGSDON, N.B. Influência da umidade nas propriedades de resistência e rigidez da madeira. 1998. 200f. Tese (Doutorado em Engenharia de Estruturas), Escola de Engenharia de São Carlos, Universidade de São Paulo, São Carlos, 1998.

MINÁ, A.J.S.; DIAS, A.A. Estacas de madeira para fundações de pontes de madeira. Cadernos de Engenharia de Estruturas, v. 10, p.129-155, 2008.

MORAES NETO, S.P.; RODRIGUES, T.O.; VALE, A.T.; SOUZA, M.R. Propriedades mecânicas da madeira de cinco procedências de Pinus caribaea var. hondurensis implantadas no cerrado do distrito federal.

Planaltina, DF: Embrapa Cerrados, 2009. (Boletim de Pesquisa e Desenvolvimento, v.251)

NASCIMENTO, H.H.C.; NOGUEIRA, R.J.M.C.; SILVA, E.C.; SILVA, M.A. Análise do crescimento

Revista Árvore, Viçosa-MG, v.40, n.1, p.147-154, 2016

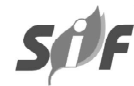


de mudas de Jatobá (Hymenaea courbaril L.) em diferentes níveis de água no solo. Revista Árvore, v.35, n3, Edição Especial, p.617-626, 2011

PAIVA, H.N.; VITAL, B.R. Escolha da espécie florestal. Viçosa, MG: Universidade Federal de Viçosa, 2003. 42p. (Cadernos Didáticos)

PLETZ, E.; ROCCO LAHR, F.A.; CALIL Jr, C.; DIAS, A.A. Análise experimental de passarela estaiada com tabuleiro de placas curvas de madeira laminada protendida. Revista

Minerva, v.3, p.113-122, 2006.

RIZZINI, C.T. Plantas do Brasil: Árvores e Madeiras Úteis do Brasil - Manual de Dendrologia
Brasileira. São Paulo: Edgard Blücher, 1978.

RODRIGUES, E.A.C.; ROSADO, S.C.S.; TRUGILLO, P.F.; SANTOS, A.M. Seleção de clones de Eucaliptus para as propriedades físicas da madeira avaliadas em árvores no campo. Revista Cerne, v. 14, n.2, p.147-152, 2008.

SIMPSON, W.T. Method to estimate drykiln schedules and species groupings: tropical and temperate hardwoods.

Madison: U. S. Department of Agriculture, Forest Service, 1996. 57p.

SOUZA, M.H.; MAGLIANO, M.M.; CAMARGOS, J.A.A. Madeiras tropicais brasileiras.

Brasília: Ministério de Meio Ambiente, 1997. 150p. 